\title{
COUNTABLE DENSE HOMOGENEOUS FILTERS AND THE MENGER COVERING PROPERTY
}

\author{
DUŠAN REPOVŠ, LYUBOMYR ZDOMSKYY, AND SHUGUO ZHANG
}

\begin{abstract}
In this note we present a ZFC construction of a non-meager filter which fails to be countable dense homogeneous. This answers a question of Hernández-Gutiérrez and Hrušák. The method of the proof also allows us to obtain a metrizable Baire topological group which is strongly locally homogeneous but not countable dense homogeneous.
\end{abstract}

\section{INTRODUCTION}

A topological space $X$ has property $C D H$ (abbreviated from countable dense homogeneous) if for arbitrary countable dense subsets $D_{0}, D_{1}$ of $X$ there exists a homeomorphism $\phi: X \rightarrow X$ such that $\phi\left[D_{0}\right]=D_{1}$. The study of CDH filters on $\omega$ was initiated in [12] where the property CDH was used to find concrete examples of non-homeomorphic filters and ultrafilters, considered with the topology inherited from $\mathcal{P}(\omega)$.

The following theorem is the main result of this note. Let us stress that we do not use any additional set-theoretic assumptions in its proof.

Theorem 1. There exists a non-meager non-CDH filter.

There are several constructions of non-CDH spaces by transfinite induction using some enumeration of all potential autohomeomorphisms, by adding points to the space under construction in such a way that these homeomorphisms are ruled out one by one, see, e.g., [12] and references therein. However, this method often requires some equalities between cardinal characteristics and hence does not seem to lead to a construction of non-meager non-CDH filters outright in ZFC.

Instead of ruling out all potential autohomeomorphisms sending some countable dense subset $D_{0}$ onto some other countable dense subset $D_{1}$ one by one, we shall do this at once. Our idea is rather straightforward: if a space $X$ admits two countable dense subsets $D_{0}, D_{1}$ such that $X \backslash D_{0}$ is not homeomorphic to $X \backslash D_{1}$, then there obviously is no autohomeomorphisms of $X$ mapping $D_{0}$ onto $D_{1}$. We shall prove Theorem 1 by constructing a non-meager filter $\mathcal{F}$ on $\omega$ and two countable dense subsets $\mathcal{D}_{0}, \mathcal{D}_{1}$ of $\mathcal{F}$ such that $\mathcal{F} \backslash \mathcal{D}_{1}$ has the Menger property whereas $\mathcal{F} \backslash \mathcal{D}_{0}$ does not, see the next section for its definition.

2010 Mathematics Subject Classification. Primary: 54D20. Secondary: 54D80, 22 A05.

Key words and phrases. CDH space, Menger space, Hurewicz space, $P$-filter, $\omega$-cover, groupable cover. 


\section{Covering properties of Menger and Hurewicz}

We recall from [15] that a topological space $X$ has

- the Menger (covering) property, if for every sequence $\left\langle\mathcal{U}_{n}: n \in \omega\right\rangle$ of open covers of $X$ there exists a sequence $\left\langle\mathcal{V}_{n}: n \in \omega\right\rangle$ such that each $\mathcal{V}_{n}$ is a finite subfamily of $\mathcal{U}_{n}$ and the collection $\left\{\cup \mathcal{V}_{n}: n \in \omega\right\}$ is a cover of $X$;

- the Hurewicz (covering) property, if for every sequence $\left\langle\mathcal{U}_{n}: n \in \omega\right\rangle$ of open covers of $X$ there exists a sequence $\left\langle\mathcal{V}_{n}: n \in \omega\right\rangle$ such that each $\mathcal{V}_{n}$ is a finite subfamily of $\mathcal{U}_{n}$ and the collection $\left\{\cup \mathcal{V}_{n}: n \in \omega\right\}$ is a $\gamma$-cover of $X$ (a family $\mathcal{U}$ of subsets of a space $X$ is called a $\gamma$-cover of $X$ if every $x \in X$ belongs to all but finitely many $U \in \mathcal{U}$ ).

These properties were introduced by Hurewicz in [8] and [9], respectively. It is clear that every $\sigma$-compact space has the Hurewicz property, and the Hurewicz property implies the Menger one. It is known [6, 10, 16] that none of these implications can be reversed. The simplest example of a metrizable space without the Menger property is the Baire space $\omega^{\omega}$. Indeed $\left\langle\mathcal{U}_{n}: n \in \omega\right\rangle$, where $\mathcal{U}_{n}=\left\{\left\{x \in \omega^{\omega}: x(n)=k\right\}: k \in \omega\right\}$, is a sequence of open covers of $\omega^{\omega}$ witnessing the failure of the Menger property.

In the proof of Theorem 1 we shall use without mentioning several basic facts about these properties summarized in the following proposition. Most likely these can be found somewhere in the literature. However, we did not try to locate them or to present their proofs as we believe that the proof of any of them should not take the reader more than a couple of minutes.

Proposition 2. (i) If a topological space $X$ has the Menger (Hurewicz) property and $Y$ is a continuous image of $X$, then $Y$ is Menger (Hurewicz);

(ii) If a topological space $X$ has the Menger (Hurewicz) property and $Y$ is a closed subspace of $X$, then $Y$ is Menger (Hurewicz);

(iii) If a topological space $X$ has the Menger (Hurewicz) property and $Y$ is compact, then $X \times Y$ is Menger (Hurewicz);

(iv) If $\left\{Y_{i}: i \in \omega\right\}$ is a collection of Menger (Hurewicz) subspaces of a space $X$, then $\bigcup_{i \in \omega} Y_{i}$ is Menger (Hurewicz);

(v) If a topological space $X$ has the Menger (Hurewicz) property and $Y$ is an $F_{\sigma}$-subspace of $X$, then $Y$ is Menger (Hurewicz);

(vi) If a topological space $X$ has the Menger (Hurewicz) property and $Y$ is $\sigma$-compact, then $X \times Y$ is Menger (Hurewicz).

Corollary 3. Let $X$ be a metrizable space with the Menger property. Then $X \backslash A$ has the Menger property for all finite subsets $A$.

Proof. $X \backslash A$ is an $F_{\sigma}$-subspace of $X$.

By a filter on a countable set $C$ we mean a free filter, i.e., a filter containing all cofinite subsets of $C$. A family $\mathcal{B} \subset \mathcal{P}(C)$ is said to be centered if for any finite $\mathcal{B}^{\prime} \subset \mathcal{B}$ the intersection $\bigcap \mathcal{B}^{\prime}$ is infinite. Any centered family generates a filter in a natural way. 
Corollary 4. Suppose that a filter $\mathcal{F}$ on a countable set $C$ is generated by a centered family $\mathcal{B}$ all of whose finite powers have the Menger property when $\mathcal{B}$ is considered with the subspace topology inherited from $\mathcal{P}(C)$. Then $\mathcal{F}$ is Menger.

Proof. Consider the map $\phi_{n}: \mathcal{B}^{n} \times \mathcal{P}(C) \times[C]^{<\omega}, \phi:\left\langle B_{0}, \ldots, B_{n-1} ; X ; x\right\rangle \mapsto$ $\left(\bigcap_{i \in n} B_{i} \backslash x\right) \cup X$. It is clear that each $\phi_{n}$ is continuous and $\mathcal{F}=\bigcup_{n \in \omega} \phi_{n}\left[\mathcal{B}^{n} \times\right.$ $\left.\mathcal{P}(C) \times[C]^{<\omega}\right]$. It suffices to use Proposition 2 several times.

\section{Proof of Theorem 1}

We shall divide the proof into two lemmas.

Lemma 5. Let $\mathcal{F}$ be a filter on $\omega$ containing co-infinite sets. Then there exists a countable dense subset $\mathcal{D}$ of $\mathcal{F}$ such that $\mathcal{F} \backslash \mathcal{D}$ does not have the Menger property.

Proof. Let us find $F \in \mathcal{F}$ such that $|\omega \backslash F|=\omega$ and consider the subspace $\mathcal{C}=\left\{F^{\prime} \in \mathcal{F}: F \subset F^{\prime}\right\}$ of $\mathcal{F}$. Let $\mathcal{D}^{\prime}, \mathcal{D}^{\prime \prime}$ be countable dense subspaces of $\mathcal{C}$ and $\mathcal{F} \backslash \mathcal{C}$, respectively. Notice that $\mathcal{C}$ is a copy of the Cantor set being compact zero-dimensional space without isolated points. Therefore $\mathcal{C} \backslash \mathcal{D}^{\prime}$ is homeomorphic to $\omega^{\omega}$. Thus $\mathcal{F} \backslash\left(\mathcal{D}^{\prime} \cup \mathcal{D}^{\prime \prime}\right)$ has a closed subspace homeomorphic to $\omega^{\omega}$ (namely $\mathcal{C} \backslash \mathcal{D}^{\prime}$ ) and hence does not have the Menger property.

A collection $\mathcal{U}$ of subsets of $X$ is called an $\omega$-cover of $X$ if $X \notin \mathcal{U}$ and for every $A \in[X]^{<\omega}$ there exists $U \in \mathcal{U}$ such that $A \subset U$. A collection $\mathcal{U}$ is a groupable $\omega$-cover of $X$ if there is a partition $\left\{\mathcal{U}_{n}: n \in \omega\right\}$ of $\mathcal{U}$ into pairwise disjoint finite sets such that for each finite subset $A$ of $X$ and for all but finitely many $n$, there exists $U \in \mathcal{U}_{n}$ such that $A \subset \mathcal{U}$.

The existence of spaces $X$ such as in the following lemma was first established in [6], see also [16, Corollary 6.4].

Lemma 6. Let $X$ be a dense subspace of $\omega^{\omega}$ such that all finite powers of $X$ have the Menger property but $X$ fails to have the Hurewicz property. Then there exists a clopen $\omega$-cover $\mathcal{V}$ of $X$ which fails to be a groupable $\omega$-cover of $X$, and such that for any two disjoint finite subsets $A, C$ of $X$ the set $\{V \in \mathcal{V}: A \subset V \wedge V \cap C=\emptyset\}$ is infinite.

Proof. Applying [11, Theorem 16] we can find a sequence $\left\langle\mathcal{U}_{n}: n \in \omega\right\rangle$ of clopen $\omega$-covers of $X$ such that for any sequence $\left\langle\mathcal{V}_{n}: n \in \omega\right\rangle, \mathcal{V}_{n} \in\left[\mathcal{U}_{n}\right]^{<\omega}$, the union $\bigcup_{n \in \omega} \mathcal{V}_{n}$ fails to be a groupable $\omega$-cover of $X$. Passing to finer $\omega$ covers, if necessary, we may additionally assume that $\mathcal{U}_{n+1}$ is a refinement of $\mathcal{U}_{n}$ for all $n$, and the projection of each element of $\mathcal{U}_{0}$ onto the 0th coordinate (recall that $\mathcal{U}_{0}$ is a family of subsets of $\omega^{\omega}$ ) is finite.

For every $s \in \omega^{<\omega}$ we shall denote the basic open subset $\left\{x \in \omega^{\omega}: x \uparrow\right.$ $|s|=s\}$ of $\omega^{\omega}$ by $[s]$. Let $\mathcal{B}=\left\{B_{k}: k \in \omega\right\}$ be the family of all finite unions of the sets of the form $[s], s \in \omega^{<\omega} \backslash\{\emptyset\}$. It follows from our restrictions on $\mathcal{U}_{n}$ 's that $\mathcal{W}_{n, k}=\left\{U \backslash B_{k}: U \in \mathcal{U}_{n}\right\}$ is an $\omega$-cover of $X \backslash B_{k}$ for all $n, k \in \omega$ (because no $U \in \mathcal{U}_{n}$ contains $X \backslash B_{k}$ ). Let us decompose $\omega$ into countably many disjoint infinite sets $\left\{I_{k}: k \in \omega\right\}$ and for every $k \in \omega$ consider the 
sequence $\left\langle\mathcal{W}_{n, k}: n \in I_{k}\right\rangle$ of clopen $\omega$-covers of $X \backslash B_{k}$. Since $X \backslash B_{k}$ is a clopen subset of $X$, all of its finite powers have the Menger property, and hence there exists a sequence $\left\langle\mathcal{V}_{n, k}: n \in I_{k}\right\rangle$ such that $\mathcal{V}_{n, k} \in\left[\mathcal{W}_{n, k}\right]^{<\omega}$ and $\bigcup_{n \in I_{k}} \mathcal{V}_{n, k}$ is an $\omega$-cover of $X \backslash B_{k}$, see [2] or [10, Theorem 3.9]. Since $\left\{X \backslash B_{k}: k \in \omega\right\}$ is an $\omega$-cover of $X$, we have that $\mathcal{V}=\bigcup_{k \in \omega, n \in I_{k}} \mathcal{V}_{n, k}$ is an $\omega$-cover of $X$. Each element of $\mathcal{V}_{n, k}$ is included in some element of $\mathcal{U}_{n}$, and hence $\mathcal{V}$ is not groupable.

Finally, let us fix some disjoint finite subsets $A, C \subset X$ and find $k \in \omega$ such that $C \subset B_{k}$ and $A \cap B_{k}=\emptyset$. Since $\bigcup_{n \in I_{k}} \mathcal{V}_{n, k}$ is an $\omega$-cover of $X \backslash B_{k}$, there are infinitely many elements of $\bigcup_{n \in I_{k}} \mathcal{V}_{n, k}$ which contain $A$. By the construction, all of them are disjoint from $C$. This completes our proof.

We are now in a position to complete the proof of Theorem 1 .

In light of Lemma 5 it is enough to construct a non-meager filter $\mathcal{F}$ and a countable dense $\mathcal{D} \subset \mathcal{F}$ such that $\mathcal{F} \backslash \mathcal{D}$ has the Menger property. Let $X$ and $\mathcal{V}$ be such as in Lemma 6. Let us fix a bijective enumeration $\left\{V_{n}: n \in \omega\right\}$ of $\mathcal{V}$ and for every $m \in \omega$ consider the mapping $f_{m}: X^{m} \rightarrow \mathcal{P}(\omega)$,

$$
f_{m}\left\langle x_{0}, \ldots, x_{m-1}\right\rangle=\left\{n:\left\{x_{0}, \ldots, x_{m-1}\right\} \subset V_{n}\right\} .
$$

Since $\mathcal{V}$ is an $\omega$-cover of $X$ we have $f_{m}\left[X^{m}\right] \subset[\omega]^{\omega}$ for all $m$. Moreover, it is easy to see that $\mathcal{Y}=\bigcup_{m \in \omega} f_{m}\left[X^{m}\right]$ is closed under finite intersections of its elements and hence it generates the filter $\mathcal{F}=\left\{F \subset \omega: Y \subset^{*} F\right.$ for some $Y \in \mathcal{Y}\}$. We claim that $\mathcal{F}$ is as required. Indeed, by Talagrand and Jalali-Naini's characterization [4, Proposition 9.4] the non-meagerness of $\mathcal{F}$ is a direct consequence of $\mathcal{V}$ not being groupable.

Let us write $\mathcal{F}$ in the form $\bigcup_{m \in \omega} \mathcal{F}_{m}$, where $\mathcal{F}_{m}=\left\{F \subset \omega: Y \subset^{*} F\right.$ for some $\left.Y \in f_{m}\left[X^{m}\right]\right\}$. Consider the map $g_{m}: X^{m} \times \mathcal{P}(\omega) \times \omega \rightarrow \mathcal{P}(\omega)$, $g_{m}(a, b, c)=\left(f_{m}(a) \backslash c\right) \cup b$. It is easy to see that $g_{m}$ is continuous and $\mathcal{F}_{m}=g_{m}\left[X^{m} \times \mathcal{P}(\omega) \times \omega\right]$. Since the Menger property is preserved by products with $\sigma$-compact spaces and continuous images, we conclude that $\mathcal{F}_{m}$ has the Menger property for all $m \in \omega$.

Now let us fix any injective sequence $\left\langle x_{m}: m \in \omega\right\rangle$ of elements of $X$ and set $F_{m}=f_{m}\left\langle x_{0}, \ldots, x_{m-1}\right\rangle$ for all $m>0$. Set also $F_{0}=\omega$. The sequence $\left\langle F_{m}: m \in \omega\right\rangle \in \mathcal{F}^{\omega}$ has the property that $\left\{F_{m}: m \in \omega\right\} \cap \mathcal{F}_{k}=\left\{F_{m}: m \in\right.$ $k+1\}$ for every $k \in \omega$. Indeed, otherwise there exists $\left\langle x_{0}^{\prime}, \ldots, x_{k-1}^{\prime}\right\rangle \in X^{k}$ such that $\phi_{k}\left\langle x_{0}^{\prime}, \ldots, x_{k-1}^{\prime}\right\rangle \subset^{*} F_{k+1}$ (because the sequence $\left\langle F_{m}: m \in \omega\right\rangle$ is decreasing). Since the sequence $\left\langle x_{m}: m \in \omega\right\rangle$ is injective, there exists $j \leq k$ such that $x_{j} \notin\left\{x_{0}^{\prime}, \ldots, x_{k-1}^{\prime}\right\}$, and hence by our choice of $\mathcal{V}$ there exist infinitely many $n \in \omega$ such that $\left\{x_{0}^{\prime}, \ldots, x_{k-1}^{\prime}\right\} \subset V_{n}$ and $x_{j} \notin V_{n}$. However, all these $n$ 's are in $\phi_{k}\left\langle x_{0}^{\prime}, \ldots, x_{k-1}^{\prime}\right\rangle$ but not in $f_{1}\left(x_{j}\right) \supset f_{k+1}\left\langle x_{0}, \ldots, x_{k}\right\rangle=$ $F_{k+1}$, a contradiction.

Since each $\mathcal{F}_{k}$ is closed under finite modifications of its elements, we can conclude that for any sequence $\left\langle F_{m}^{\prime}: m \in \omega\right\rangle \in \mathcal{F}^{\omega}$, if $F_{m}={ }^{*} F_{m}^{\prime}$ for all $m$, then $\left\{F_{m}^{\prime}: m \in \omega\right\} \cap \mathcal{F}_{k}=\left\{F_{m}^{\prime}: m \in k+1\right\}$ for all $k$.

Let $\left\{s_{m}: m \in \omega\right\}$ be an enumeration of $2^{<\omega}$ and $F_{m}^{\prime}=\left[F_{m} \backslash\left(\operatorname{dom}\left(s_{m}\right)\right)\right] \cup$ $s_{m}^{-1}(1)$. It is clear that $\mathcal{D}=\left\{F_{m}^{\prime}: m \in \omega\right\}$ is dense in $\mathcal{F}$. It follows from 
the above that

$$
\mathcal{F} \backslash \mathcal{D}=\bigcup_{k \in \omega} \mathcal{F}_{k} \backslash \mathcal{D}=\bigcup_{k \in \omega}\left(\mathcal{F}_{k} \backslash\left\{F_{m}^{\prime}: m \in k+1\right\}\right) .
$$

Since each $\mathcal{F}_{k}$ has the Menger property, by Corollary 3 we have that $\mathcal{F} \backslash \mathcal{D}$ is a countable union of its subspaces with the Menger property, and hence itself has the Menger property. This completes our proof.

A space $X$ is called strongly locally homogeneous if it has an open base $\mathcal{B}$ such that, for each $U \in \mathcal{B}$ and points $x, y \in U$, there exists a homeomorphism $h: X \rightarrow X$ with $h(x)=y$ and $h \uparrow(X \backslash U)$ equal to the identity. It has been shown in [1] that every strongly locally homogeneous Polish space is CDH. This result is not anymore true for Baire spaces, even in the realm of separable metrizable spaces: for every $n \in \omega \cup\{\infty\}$ there exists an $n$-dimensional Baire space which is strongly locally homogeneous but not CDH, see [13, Remark 4.1].

However, spaces constructed in [13] are not topological groups, see Theorem 3.5 there. Thus the filter $\mathcal{F}$ constructed in the proof of Theorem 1 seems to be the first example of a metrizable separable Baire topological group which is strongly locally homogeneous but not $\mathrm{CDH}$. It might be also worth noticing that it cannot be made $\mathrm{CDH}$ by products with metrizable compact spaces.

Proposition 7. Let $\mathcal{F}$ be the filter constructed in the proof of Theorem 1 . Then $\mathcal{F} \times Y$ is not $C D H$ for any metrizable compact $Y$.

Proof. We shall use notation from the proof of Theorem 1. Let $\left\langle y_{m}: m \in \omega\right\rangle$ be a sequence of elements of $Y$ such that $\mathcal{D}_{p}:=\left\{\left\langle F_{m}^{\prime}, y_{m}\right\rangle: m \in \omega\right\}$ is dense in $\mathcal{F} \times Y$. Since $\mathcal{F}_{k} \cap\left\{F_{m}^{\prime}: m \in \omega\right\}$ is finite for all $k \in \omega$, we have that $\left(\mathcal{F}_{k} \times Y\right) \cap \mathcal{D}_{p}$ is finite for all $k \in \omega$. Since $\mathcal{F}_{k}$ is Menger, so is $\mathcal{F}_{k} \times Y$, and hence $\left(\mathcal{F}_{k} \times Y\right) \backslash \mathcal{D}_{p}$ is Menger as well. Therefore $(\mathcal{F} \times Y) \backslash \mathcal{D}_{p}=$ $\bigcup_{k \in \omega}\left(\mathcal{F}_{k} \times Y\right) \backslash \mathcal{D}_{p}$ is also Menger. Thus $\mathcal{F} \times Y$ has a countable dense subset with Menger complement.

On the other hand, the same argument as in the proof of Lemma 5 implies that $\mathcal{F} \times Y$ has a countable dense subset whose complements in $\mathcal{F} \times Y$ is not Menger. This completes our proof.

We call a filter $\mathcal{F}$ on $\omega$ a $P^{+}$-filter, if for every sequence $\left\langle A_{n}: n \in \omega\right\rangle$ of elements of $\mathcal{F}^{+}=\{X \subset \omega: \forall F \in \mathcal{F}(X \cap F \neq \emptyset)\}$ there is a sequence $\left\langle B_{n}: n \in \omega\right\rangle$ such that $B_{n} \in\left[A_{n}\right]^{<\omega}$ and $\bigcup_{n \in \omega} B_{n} \in \mathcal{F}^{+}$. Replacing $\mathcal{F}^{+}$ with $\mathcal{F}$ in the definition above we get the classical notion of a $P$-filter. Every filter with the Menger property is a $P^{+}$-filter. Indeed, the Menger property applied to the collection $\left\{\mathcal{U}_{A}: A \in \mathcal{F}^{+}\right\}$of open covers of $\mathcal{F}$, where $\mathcal{U}_{A}=\{\{X \subset \omega: n \in X\}: n \in A\}$, gives nothing else but the definition of $P^{+}$-filters. If $\mathcal{F}$ is an ultrafilter then $\mathcal{F}^{+}=\mathcal{F}$ and hence $\mathcal{F}$ is a $P$-filter if and only if it is a $P^{+}$-filter. Since the non-meager non-CDH filter constructed in the proof of Theorem 1 has the Menger property, we get the following

Corollary 8. There exists a non-meager $P^{+}$-filter which is not CDH. 
Corollary 8 follows directly from Theorem 11, and hence its proof does not require anything beyond ZFC. Corollary 8 implies that one of the main results of [7] which states that non-meager $P$-filters are $\mathrm{CDH}$ is sharp in the sense that it cannot be extended to $P^{+}$-filters, even under additional set-theoretic assumptions.

Following [3] we call filters $\mathcal{F}_{0}$ and $\mathcal{F}_{1}$ on $\omega$ coherent, if there exists a monotone surjection $\psi: \omega \rightarrow \omega$ such that $\psi\left[\mathcal{F}_{0}\right]=\psi\left[\mathcal{F}_{1}\right]$. It is easy to see that the coherence is an equivalence relation. It has been shown in [5] that in the model constructed by Miller in [14, any two non-meager filters are coherent and there exists a $P$-point, i.e., an ultrafilter which is a $P$-filter. Together with Theorem 1 this proves the following

Corollary 9. In the Miller model the collection of all CDH filters is not closed under the coherence relation.

We do not know whether Corollary 9 is true in ZFC. Let us note that a ZFC proof of it would require at least a construction of a CDH filter without any additional set-theoretic assumption, which seems to be quite a difficult task.

We recall that $\mathfrak{d}$ is, by definition, the minimal cardinality of a cover of $\omega^{\omega}$ by its compact subspaces, and $\mathfrak{u}$ is the minimal cardinality of a base of an ultrafilter on $\omega$. We refer the reader to [4] for more information on $\mathfrak{u}, \mathfrak{d}$, and other cardinals characteristics of the continuum.

Remark. In the proof of Theorem 1 we had to be rather careful with the choice of $\mathcal{V}$. This is because the same argument would not work if we started with $\mathcal{V}$ which satisfies all requirements of Lemma 6 except for the last one, i.e., does not allow to sufficiently distinguish between disjoint finite subsets of $X$. Indeed, assume $\mathfrak{u}<\mathfrak{d}$, which holds, e.g., in the aforementioned Miller model, and let $\mathcal{U}$ be an ultrafilter with $\mathfrak{u}$-many generators. Then $\mathcal{U}$ fails to have the Hurewicz property by [10, Theorem 4.3] being a non-meager subset of $[\omega]]^{\omega}$. On the other hand, by [10, Theorem 4.4] all finite powers of $\mathcal{U}$ have the Menger property because $\mathcal{U}$ is a union of fewer than $\mathfrak{d}$ compact spaces. Now set $\mathcal{V}=\left\{V_{n}: n \in \omega\right\}$, where $V_{n}=\{U \in \mathcal{U}: n \in U\}$. It is easy to check that $\mathcal{V}$ is an $\omega$-cover of $\mathcal{U}$ which fails to be groupable. Letting $X=\mathcal{U}$ and defining $\phi_{m}$ 's and $\mathcal{F}$ in the same way as in the proof of Theorem 1, one can easily check that $\mathcal{F}=\mathcal{U}$. However, $\mathcal{U}$ is a $P$-point, see, e.g., [4, Theorem 9.25]. Therefore $\mathcal{U}$ is $\mathrm{CDH}$ [7] and hence by Lemma 5 it is impossible to select a countable dense $\mathcal{D} \subset \mathcal{U}$ such that $\mathcal{U} \backslash \mathcal{D}$ has the Menger property.

Acknowledgment. The second author would like to thank M. Hrušák and S. Todorčević for useful discussions during the Winter School in abstract Analysis (Set Theory and Topology Section) held in Hejnice in January 2013. Also, we are grateful to A. Medini for bringing the paper [13] to our attention and for detecting some inaccuracies in the previous versions.

The first author acknowledges the support of the ARRS grant P1-02920101. The second author would like to thank the Austrian Academy of Sciences (APART Program) as well as the Austrian Science Fund FWF 
(Grants M 1244-N13 and I 1209-N2) for generous support for this research. Parts of the work reported here were carried out during the visit of the third named author at the Kurt Gödel Research Center in November 2012. This visit was supported by the above-mentioned FWF grant. The third author thanks the second one for his kind hospitality. The third author would also like to acknowledge the support of the NSFC grant \#11271272.

\section{REFERENCES}

[1] Anderson, R. D.; Curtis, D. W.; van Mill, J., A fake topological Hilbert space, Trans. Amer. Math. Soc. 272 (1982), 311-321.

[2] Arkhangel'skii, A.V., Hurewicz spaces, analytic sets and fan tightness of function spaces, Dokl. Akad. Nauk SSSR 287 (1986), 525-528. (In Russian)

[3] Banakh, T.; Zdomskyy, L., Coherence of Semifilters, book in preparation. http://www.franko.lviv.ua/faculty/mechmat/Departments/Topology/booksite.html.

[4] Blass, A., Combinatorial cardinal characteristics of the continuum, in: Handbook of Set Theory (M. Foreman, A. Kanamori, and M. Magidor, eds.), Springer, 2010, pp. 395-491.

[5] A. Blass, C. Laflamme, Consistency results about filters and the number of inequivalent growth types, J. Symbolic Logic 54 (1989), 50-56.

[6] Chaber, J.; Pol, R., A remark on Fremlin-Miller theorem concerning the Menger property and Michael concentrated sets, unpublished notes, 2002.

[7] Hernández-Gutiérrez, R.; Hrušák, M., Non-meager P-filters are countable dense homogeneous, Colloq. Math. 130 (2013), 281-289.

[8] Hurewicz, W., Über die Verallgemeinerung des Borellschen Theorems, Math. Z. 24 (1925), 401-421.

[9] Hurewicz, W., Über Folgen stetiger Funktionen, Fund. Math. 9 (1927), 193204.

[10] Just, W.; Miller, A.; Scheepers, M.; Szeptycki, P., The combinatorics of open covers (II), Topology Appl. 73 (1996), 241-266.

[11] Kočinac, L.D.R.; Scheepers, M., Combinatorics of open covers. VII. Groupability, Fund. Math. 179 (2003), 131-155.

[12] Medini, A.; Milovich, D., The topology of ultrafilters as subspaces of $\mathcal{P}(\omega)$, Topology Appl. 159 (2012), 1318-1333.

[13] Van Mill, J., Strong local homogeneity does not imply countable dense homogeneity, Proc. Amer. Math. Soc. 84 (1982), 143-148.

[14] A. Miller, Rational perfect set forcing, in: Axiomatic Set Theory (J. Baumgartner, D. A. Martin, S. Shelah, eds.), Contemporary Mathematics 31, American Mathematical Society, Providence, Rhode Island, 1984, pp. 143-159.

[15] Scheepers, M., Combinatorics of open covers. I. Ramsey theory, Topology Appl. 69 (1996), 31-62.

[16] Tsaban, B., Zdomskyy, L., Scales, fields, and a problem of Hurewicz, J. Eur. Math. Soc. 10 (2008), 837-866.

Faculty of Education, and Faculty of Mathematics and Physics, University of Luubluana, P. O. Box 2964, Ljubluana, Slovenia 1001.

E-mail address: dusan.repovs@guest.arnes.si

$U R L:$ http://www.fmf .uni-lj.si/〜repovs/index.htm

Kurt Gödel Research Center for Mathematical Logic, University of Vienna, Währinger Strasse 25, A-1090 Wien, Austria.

E-mail address: lzdomsky@gmail.com

$U R L:$ http://www.logic.univie.ac.at/〜 lzdomsky/ 
College of Mathematics, Sichuan University, Chengdu, Sichuan 610064, CHINA.

E-mail address: zhangsg@scu.edu.cn 